\title{
A Modified Wavelet-Common Spatial Pattern Method for Decoding Hand Movement Directions in Brain Computer Interfaces
}

\author{
Neethu Robinson, A. P. Vinod \\ School of Computer Engineering \\ Nanyang Technological University \\ Singapore \\ neethu1@e.ntu.edu.sg
}

\author{
Cuntai Guan, Kai Keng Ang, Tee Keng Peng \\ Institute for Infocomm Research \\ Agency for Science, Technology and Research \\ Singapore
}

\begin{abstract}
The decoding of hand movement kinematics using noninvasive data acquisition techniques is a recent area of research in Brain Computer Interface (BCI). In this work, we use an Electroencephalography (EEG) based BCI to decode directional information from the brain data collected during an actual hand movement experiment. The objective is to find the discriminative features of movement related potential that can classify any two directions out of the four orthogonal directions in which subject performs right hand movement. The performance using Wavelet-Common Spatial Pattern (W-CSP) algorithm and its variations in terms of spatial regularization is studied and compared. The work further analyzes the involvement of frontal, parietal and motor regions in carrying movement kinematics information with the help of spatial plots given by CSP. The performance variability for different directions in various subjects is another important observation in our results. The work aims to provide a more refined movement control command set for BCIs by developing efficient techniques to decode the direction of movement.
\end{abstract}

KeywordsBrain

Computer

Electroencephalography; Common Spatial Pattern; Spatial Regularization; Discrete Wavelet Transform.

\section{INTRODUCTION}

A Brain Computer Interface (BCI) based on electroencephalography (EEG) provides a non-invasive technique to acquire brain activity and further translate this activity into control commands for an external device [1]. The movement control command set available for the BCI is limited due to lack of knowledge about the discriminative features regarding the details of movement namely the limb position, speed, direction and acceleration. Contrary to the belief that non-invasive scalp recordings do not contain sufficient signal-to-noise ratio (SNR) or spatial resolution to provide movement parameter information, recent studies using various non-invasive modalities such as EEG, Magnetoencephalography (MEG) and Near Infrared Spectroscopy (NIRS) shows the possibilities of decoding hand movement kinematics from brain signals [2]. A work using MEG based BCI that classified various directions of wrist movement using various signal processing and classification techniques was reported in [3]. Another work reported in [4] aimed to discriminate the direction of arm force using brain signal recorded by NIRS. The work in [5] used the EEG data recorded during a delayed saccade or reach task and decoded intended movement direction by analyzing multi channel Event Related Potential (ERP) using Independent Component Analysis (ICA). In [6], authors attempted to decode hand movement direction from EEG which used normalized variances of CSP-spatially filtered EEG signals as features for for classifying orthogonal directions.

Various works have been done on decoding and reconstruction of hand movement kinematics using EEG based $\mathrm{BCI}$ and certain works have mentioned the features related to movement kinematics [2, 7,8$]$. In [7], authors reported that the frequency range that provided best movement direction information was the low frequency (LF) range of less than 7 Hz. The study reported in [2] using MEG showed that the 2-5 $\mathrm{Hz}$ EEG frequency components provided the movement kinematics information, which substantiates the findings in [7]. A study based on partially invasive Electrocorticography in [8] reported that significant direction related information was present in the premotor, lateral prefrontal and posterior parietal regions of the brain and the best discriminative features were derived from the LF bands. Using these information, we proposed an algorithm to analyze features from LF movement related potentials of brain signals to derive directional information and was reported in [9]. Based on the requirements of localization in spectro-tempero-spatial domains, the technique used wavelets to construct an orthogonal filter bank which localized signals spectro-temporally and CSP which subsequently spatially filter the signals. An average classification accuracy of $87.85 \%$ for two subjects was reported in [9].

The objective of our work is to decode directional information from LF movement related potential recorded from brain using EEG. In the current work, we modify our Wavelet-Common Spatial Pattern (W-CSP) algorithm in [9] to improve its direction-decoding accuracy. We limited our analysis by using wavelet coefficients from the LF region which carries more directional information. In this work, we perform an experiment with the objective of acquiring data during actual hand movement in four different directions similar to the one reported in [9]. The performance of the WCSP algorithm in [9] is determined and it is found to degrade 
in some subjects. To reason the degradation, we have performed a feature analysis and have found the existence of CSP-over fitting problem. CSP being very sensitive to noise and outliers, tends to get trained favoring the noise in the training data set which leads to over fitting. The over fitting problem has been solved using various methods in literature [10-12]. In our modified algorithm, we perform a Spatial Regularized CSP (SR-CSP) reported in [10] which penalizes spatially non-smooth filters and thus avoids over-fitting of CSP. It considers spatial location of the electrodes to construct spatial filters. In our experiment, electrodes covering both hemispheres are included in the analysis to obtain better spatial information.

This paper is organized as follows: Section II describes the feature extraction algorithms employed in this work. Section III describes experiment protocol and data analyses performed. Section IV presents our results and the discussions followed by Section V which concludes the work.

\section{FEATURE EXTRACTION TECHNIQUES}

Our objective is to determine the features in the LF band of movement related potential recorded by EEG and apply these discriminative features for classifying direction. This requires the decomposition of LF band into various subbands followed by spatial filtering. There are various methods for the spectral and spatial analysis including Filter Bank CSP (FBCSP) [13] and its variants. The design of narrowband filters for splitting the LF EEG band into subbands is not a practically viable solution especially when high subband resolution is desired. Therefore, taking into account the requirements of time localization and high resolution in low frequency bands, wavelets are used in the W-CSP algorithm to construct an orthogonal filter bank instead of conventional filters. In this section, we present the modified W-CSP algorithm that improves the classification accuracy of the W-CSP algorithm in [9].

The block diagram in Fig. 1 presents the proposed modified W-CSP algorithm. It consists of a data acquisition and preprocessing stage followed by a feature extraction stage. The feature extraction comprises of wavelet decomposition and the wavelet coefficients were spatially filtered using SR-CSP. The features thus extracted are given to the classifier. The detailed analysis is as follows:

\section{A. $W-C S P$}

The feature extraction procedure using W-CSP [9] is as follows. The preprocessed EEG data is given as its input. The initial step is to break down the signal into various subbands using wavelets, which is an effective way to analyze non stationary signals [15]. The discrete wavelet transform (DWT) is used which performs multiresolution analysis on the signal and decomposes it into coarse approximation and detailed information. Wavelets act as band pass filters, whose contracted version (high frequency) performs fine temporal analysis and dilated version (low frequency) performs fine frequency analysis [14].

Let the single trial pre processed EEG be represented as $X$ $=\left[\boldsymbol{x}_{1}, \boldsymbol{x}_{2}, \ldots \boldsymbol{x}_{N}\right]$, where $\boldsymbol{x}_{i}$ corresponds to $T$-length sequence from $i^{t h}$ channel and $N$ is the total number of channels. The
DWT requires two orthonormal functions spanning non overlapping sub spaces, which effectively performs lowpass and highpass filtering of the signal. These functions are denoted by $h_{0}$ and $h_{l}$, where $h_{0}$ and its even shifted version $h_{l}$ correspond to the lowpass filter and highpass filter respectively. The wavelet decomposition thus involves half band lowpass and highpass filtering followed by down sampling by 2 and it produces Approximation $(C A)$ and Decomposition $(C D)$ wavelet coefficients of the signal respectively. The W-CSP algorithm in [9] uses wavelet decomposition in a scaled to zero approach. This means it decomposes signal into its maximum possible number of levels of decomposition. For a $T$-sample sequence this is given by $J \max =\log 2(T)$. The coefficients at each level are obtained as $\left\{C D_{1}, C D_{2} \ldots C D_{\text {Jmax }}, C A_{\text {Jmax }}\right\}$ which correspond to various subbands.

At each level, the signals are reconstructed using the time reversed impulse responses. Let $Y_{j}$ be the reconstructed signal in $j^{\text {th }}$ level, where $Y_{j}=\left[\boldsymbol{y}_{j 1}, \boldsymbol{y}_{j 2}, \ldots \boldsymbol{y}_{j N}\right]$, where $\boldsymbol{y}_{\boldsymbol{j} i}$ represents $T$ length reconstructed signal for $i^{\text {th }}$ channel, $(i=1$ to $N)$ in $j^{\text {th }}$ sub band, $(j=1$ to $\operatorname{Jmax}+1)$. Thus we obtain the temporally localized signals at various subbands. The subband signals are spatially filtered using the well known CSP algorithm [16] which obtains the most discriminative features between two classes. For subband $j$, the CSP algorithm aims to obtain a spatial filter $W_{j}$ which extremizes the function given in (1)

$$
J\left(W_{j}\right)=\frac{W_{j}^{T} Y_{j 1}^{T} Y_{j 1} W_{j}}{W_{j}^{T} Y_{j 2}^{T} Y_{j 2} W_{j}}=\frac{W_{j}^{T} C_{j 1} W_{j}}{W_{j}^{T} C_{j 2} W_{j}}
$$

where $Y_{j 1 / 2}$ is the reconstructed data matrix for class 1 and 2 and $C_{j}{ }_{1 / 2}$ are the spatial co variances of the respective classes. This optimization problem is solved using joint diagonalization of matrices $C_{j 1}$ and $C_{j 2}$ to obtain spatial filter $W_{j}$ and the spatially filtered signal as $Z_{j}=W_{j} . Y_{j}$. The logarithm of normalized variances of $Z_{j}$ are used as the features in that band.

\section{B. Proposed modified W-CSP algorithm}

Spatial Regularization for CSP: As discussed in Section I, a major drawback of W-CSP algorithm is that its performance in certain subjects was found to degrade and feature space analysis of the data showed presence of over-fitting of CSP. An effective solution for this issue, SR-CSP in [10] which uses the spatial information of the electrodes to regularize the CSP. This algorithm penalized the filters which are not spatially smooth by adding a penalty term to the classical CSP equation (1). We incorporate the SR-CSP approach in the decomposed subband coefficients obtained from wavelet decomposition and the steps are given below. For subband $j$, the objective function in (1), when regularized takes the form,

$$
J_{1}\left(W_{j}\right)=\frac{W_{j}^{T} C_{j 1} W_{j}}{W_{j}^{T} C_{j 2} W_{j}+\alpha \cdot P\left(W_{j}\right)}
$$

Here $P\left(W_{j}\right)$ measure the spatial smoothness of $W_{j}$ and this term needs to be minimized for increasing smoothness of the filter. $\alpha$ is a positive constant which defines level of smoothness. $P\left(W_{j}\right)$ is defined as in (3),

$$
P\left(W_{j}\right)=W_{j}^{T}(D-G) W_{j}
$$


where $G$ is a Gaussian kernel, $G_{a b}=\exp \left(-\frac{\left\|v_{a}-v_{b}\right\|^{2}}{2 r^{2}}\right)$ and $v_{a / b}$ are spatial coordinates of electrodes $a$ or $b$. Here the hyper parameter $r$ defines the maximum distance between two electrodes that are considered close to each other. Thus the objective function is modified as,

$$
J_{1}\left(W_{j}\right)=\frac{W_{j}^{T} C_{j 1} W_{j}}{W_{j}^{T}\left(C_{j 2}+\alpha \cdot K\right) W_{j}}
$$

Similarly we define the counterpart of (4) for class 2 and these are analyzed using Lagrange multiplier method. The solution is obtained as,

$$
M_{j 1}=\left(C_{j 2}+\alpha k\right)^{-1} C_{j 1}, M_{j 2}=\left(C_{j 1}+\alpha k\right)^{-1} C_{j 2}
$$

To maximize each of these objective functions, the eigen vectors corresponding to the largest eigen values of $M_{j 1}$ and $M_{j 2}$ as spatial filters $W_{j}$ are taken.

In the proposed modified W-CSP algorithm, instead of using all Jmax subbands, we choose Jnew $<(\max +1)$ subbands which correspond to the LF band. The coefficients from Jnew subbands, are spatially filtered using the mentioned spatially regularized CSP algorithm.

The data analysis algorithm in Fig. 1 can be consolidated in the following steps:

Step 1: The EEG data acquired, is pre-processed which includes base line correction and filtering.

Step2: The signal is decomposed into various subbands using wavelet decomposition.

Step 3: The wavelet coefficients in Jnew selected subbands are applied to spatially regularized CSP algorithm that performs spatial filtering to extract the most discriminative features.

Step 4: Classification of features is performed using Fisher Linear Discriminant (FLD) classifier [17] and performance is obtained in terms of mean classification accuracy for a 10-fold cross validation.

\section{EXPERIMENT AND DATA ANALYSIS}

In order to decode directional information from movement related LF potential of EEG, an experiment protocol is developed to acquire brain signals while subjects performed right hand movement in four different specified directions. This section describes the experimental set up and the analysis performed.

\section{A. Subject and Equipments}

The experiment was performed in the Neural Signal Processing Lab of Institute of Infocomm Research, Agency for Science, Technology and Research, Singapore. The data was recorded using Neuroscan SynAmps 128 channel EEG amplifier. MIT MANUS robot used during data collection recorded the exact hand position at every sample time. The subjects (seven healthy right handed males) were asked to perform the experiment as given in the protocol. The data were recorded from 118 channels all over the scalp and for analysis,
35 channels spanning frontal, central and parietal regions were used.

\section{B. Protocol}

The subjects required to perform hand movement directions as shown in Fig. 2. The movements were performed in horizontal plane and North, South, East and West represents movement away from the body, towards the body, to the right and left respectively. The timeline is also shown in Fig. 2-i and the steps are as follows.

The subject was seated on a chair with arm resting on a table, facing a computer monitor, which provided the visual cue and feedback. Fig. 2-ii shows the visual provided for the task 'North', or hand movement in forward direction. At the start of trial, a home screen (a) was shown with encircled cross. The target cue (circle in one of four directions) was displayed at the end of the rest period (b). The target location can be any of the four directions -North, South, East and West. At the end of preparation interval, the circle disappeared, signaling the start of $\operatorname{task}(\mathrm{c})$. The task duration was around 3 seconds, at the end of which cross appeared at the target (d). In the following 2 seconds, a feedback for the user was provided in which a successful trial was indicated by reappearance of the circle at the rest position (e). The display returns to home screen and this cycle was repeated.

During the task interval, subject was asked to move the right hand in a horizontal plane in the specified direction. The subject held a MIT MANUS robot in his hand while the task was performed. This device recorded the hand position at every sample time. A cycle of trials containing all 4 directions in randomized order, was defined. Thus in each cycle, four trials of data were recorded. Each session of experiment consisted of 50 cycles and 2 sessions were performed by each subject. Each trial took 10 seconds and thus the time taken to record data from each subject was around 1 hour 26 minutes.

\section{Analysis}

The preprocessing steps include baseline correction, low pass filtering at $100 \mathrm{~Hz}$ and notch filtering at $50 \mathrm{~Hz}$. The time segment extracted from each trial includes the movement preparation (planning) and the movement execution period. The preprocessing is followed by our modified W-CSP algorithm. The extracted features were classified using a FLD classifier. It provides a linear discriminant that maximizes the ratio of between class scatter to within class scatter. Binary classification is performed taking only 2 classes at a time. The detailed results are given in the following section along with the inferences.

\section{RESULTS AND DISCUSSIONS}

\section{A. Performance of Algorithms in Direction Classification}

The decoding performance of various algorithms is reported in Table I and the various conclusions regarding the results are given below. The entries given in bold are the maximum classification accuracy obtained for each of the classifications.

1) $W$-CSP: The method in [9] was performed on the data acquired. The detailed results show that out of seven subjects, 
three subjects perform very poorly in all the classifications which deteriorates the average performance.

2) Spatial regularization for $W$-CSP: The proposed modification on W-CSP is used for extracting features from the data. The parameters required for the algorithm were manually set as $\alpha=(10 e-4)$ and $r=1$. The results obtained are as shown in Table I and an utmost improvement of around $4.8 \%$ (for D1-D4) is obtained in performance and an average increase of $2.53 \%$ is obtained.

\section{B. Spatial Patterns for Activation}

The CSP patterns derived during our analysis show clear activation in frontal, parietal and motor regions at LF bands. Fig. 3 shows the patterns for Jnew $=6$ levels used for classification North v/s East (D1-D2). This validates the role of frontal, parietal and motor regions in carrying directional information, discussed in literature.

\section{Subject Specific Variation in Performance}

Our results using the modified W-CSP approach showed a variation in performance for different subjects for different classifications. The results are given in the Table II. The best performance for each subject is given in bold. This may indicate the difference in which each subject plans or performs the movement which provides distinguishable features in a particular direction.

\section{CONCLUSION}

The paper reports our work on directional decoding of EEG data collected during an actual hand movement. The performance of a W-CSP algorithm was analyzed and was compared against its proposed variant. The modified W-CSP algorithm in terms of spatial regularization improved the classification accuracy on an average. Moreover, the algorithm used LF MRP features for classifying direction and the spatial patterns were analyzed using common spatial patterns. The results prove that the low frequency signal features from frontal, parietal and motor regions play key role in direction decoding and these conclusions agree with the existing information on spatial and spectral activation of movement kinematics. The results obtained indicate the possibility of decoding direction of hand movement using the non-invasive EEG recordings.

Our results indicated the possibilities of decoding movement kinematics from scalp recordings. This leads to inclusion of more commands in a BCI system for movement control. The future work includes the introduction of multi class classifiers which considers multiple directions at a time for discrimination. Also, the algorithm introduced demands optimization in terms of subject dependent parameter selection. Last but not the least, as every study in SMR-based BCI, this study also aims to aid in rehabilitation of stroke patients. As the overlap of features between actual movement and motor imagery is high, this study could provide some of the features for dealing with motor imagery signals too. The results obtained show a possibility in expanding this area of BCI research in terms of further improvement both in performance and movement control command set.

\section{REFERENCES}

[1] J. R. Wolpaw, N. Birbaumer, D. J. McFarland, G. Pfurtscheller, T. M. Vaughan, "Brain-computer interfaces for communication and control," Clinical Neurophysiology, vol. 113, no. 6, pp. 767-791, June 2002.

[2] K. Jerbi, J. R. Vidal, J. Mattout, F. Lecaignard, T. Ossandon, C. M. Hamame, et al., "Inferring hand movement kinematics from MEG, EEG and intracranial EEG: From brain-machine interfaces to motor rehabilitation, " IRBM, vol. 32, no. 1, pp. 8-18, Feb. 2011.

[3] N. I. Sabra, M. A. Wahed, "The use of MEG-based brain computer interface for classification of wrist movements in four different directions", $28^{\text {th }}$ National Radio Science Conference, pp. 1-7, Cairo, Egypt, April 2011,.

[4] T. Sato, T. Tsubone, Y. Wada, "Estimation of the direction of arm force by using NIRS signals," $31^{\text {st }}$ Annual International Conference of the IEEE EMBS, pp. 590-593, Minneapolis, Minnesota, USA, Sep. 2009.

[5] Y. Wang, S. Makieg, "Predicting intended movement direction using EEG from human posterior parital cortex, "Lecture notes in Computer Science, $5^{\text {th }}$ International Conference on Foundations of Augmented Cognition, Neuroergonomics and Operational Neuroscience, pp. 437446, San Diego, CA, July 2009.

[6] G. Clauzel, C. Neuper, G.M-Putz, "Offline decoding of hand movement directions from non-invasive EEG," International Journal of Bioelectromagnetism, vol.13, no. 2, pp. 90-91, 2011.

[7] S. Waldert, T. Pistohl, C. Braun, T. Ball, A. Aertsen, "A review on directional information in neural signals for brain-machine interfaces," Journal of Physiology (Paris), vol. 103, no. 3-5, pp. 244-254, Aug. 2009.

[8] T. Ball, A. S-Bonhage, A. Aertsen, C. Mehring, "Differential representation of arm movement direction in relation to cortical anatomy and function," J. Neural Eng., vol. 6, no. 1, pp.68-73, Feb. 2009.

[9] Neethu. R., A. P. Vinod, C. Guan, K. K. Ang, K. P. Tee, "A WaveletCSP method to classify hand movement directions in EEG based BCI system," Proc. of $8^{\text {th }}$ International Conference on Information, Communications and Signal Processing, Accepted for publication, Singapore, Dec. 2011.

[10] F. Lotte, C. Guan, "Spatially regularized common spatial patterns for EEG classification," $20^{\text {th }}$ International Conference on Pattern Recognition, vol. 2, no. 1, pp. 3712-3715, Istanbul, Turkey, Aug. 2010.

[11] F. Lotte, C. Guan, "Regularizing common spatial patterns to improve BCI designs: Unified theory and new algorithms", IEEE Transactions on Biomedical Engineering, vol.58, no.2, pp. 355-362, Feb. 2011.

[12] Z. Xiang, Y. Xi, U. Hasson, P. Ramadge, "Boosting with spatial regularization", $23^{\text {rd }}$ Annual Conference on Neural Information Processing Systems, pp. 2107-2115, Vancouver, Canada, Dec. 2009.

[13] K. K. Ang, Z. Y. Chin, H. Zhang, C. Guan, "Filter bank common spatial pattern (FBCSP) in brain-computer interface," Proc. of IEEE Joint Conference on Neural Networks, pp. 2390-2397, Hongkong, June 2008.

[14] M. Vetterli, C. Herley, "Wavelets and filter banks: Theory and design," IEEE Transactions in Signal Processing, vol. 40, no. 9, pp. 2207-2232, Sep. 1992.

[15] S. Mallat, "A wavelet tour of signal processing," Academic Press, CA, $2^{\text {nd }}$ ed., 1999.

[16] H. Ramoser, J. M-Gerking, G. Pfurtscheller, "Optimal spatial filtering of single trial EEG during imagined hand movement," IEEE Transactions on Rehabilitation Engineering, vol. 8, no. 4, pp. 441-446, Dec. 2000.

[17] R. O. Duda, P. E. Hart, D. G. Stork, “Pattern Classification,” New York: Wiley, $2^{\text {nd }}$ ed., 2001. 


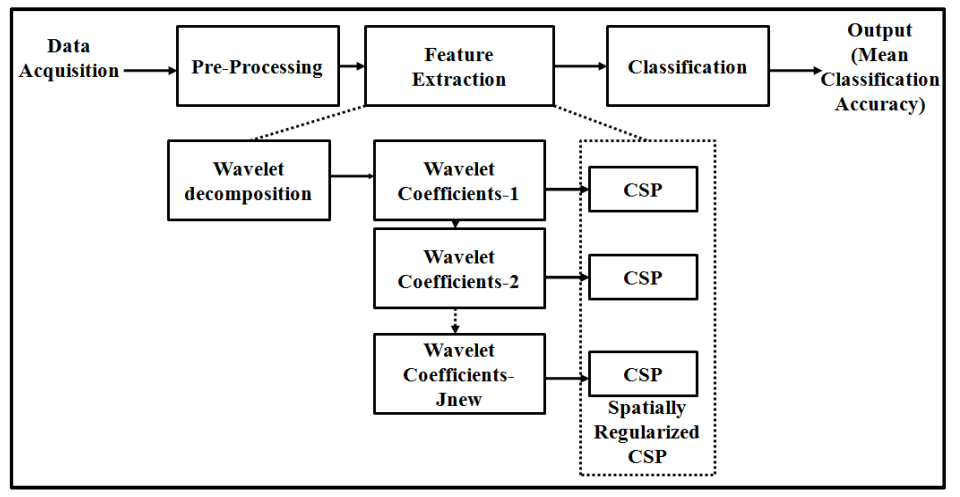

Figure 1. Proposed feature extraction technique.

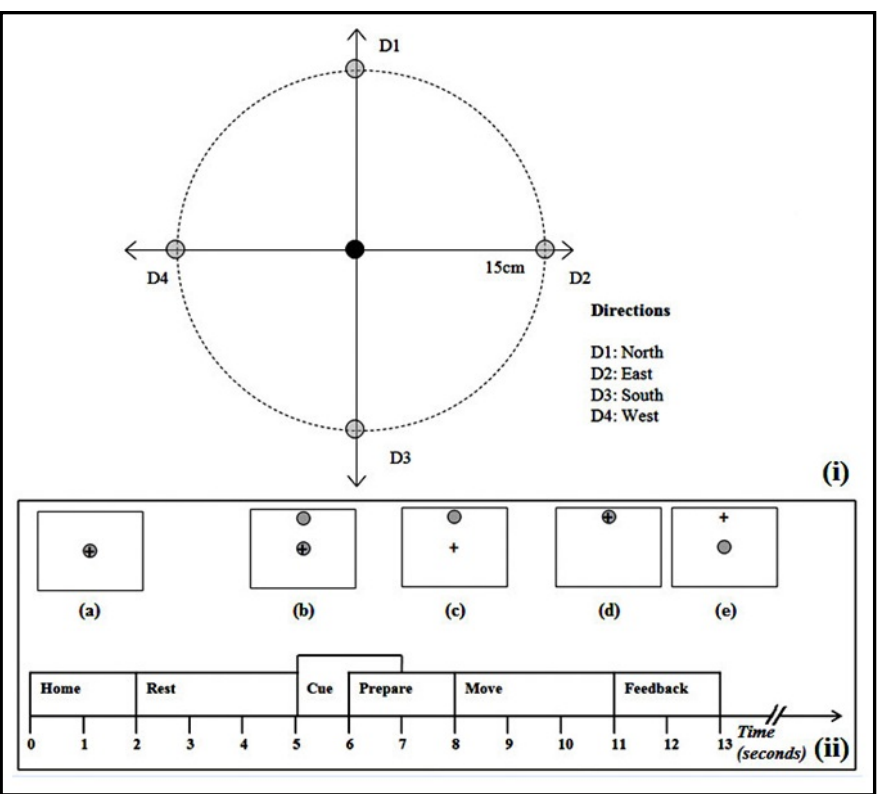

Figure 2. Experiment timeline and protocol.
TABLE I. COMPARING PERFORMANCE IN TERMS OF MEAN CLASSIFICATION ACCURACY OF VARIOUS TECHNIQUES (IN PERCENTAGE).

\begin{tabular}{|c|c|c|c|c|c|c|c|}
\hline $\begin{array}{c}\text { Class versus } \\
\text { Method }\end{array}$ & $\begin{array}{c}\text { D1- } \\
\text { D2 }\end{array}$ & $\begin{array}{c}\text { D1- } \\
\text { D3 }\end{array}$ & $\begin{array}{c}\text { D1- } \\
\text { D4 }\end{array}$ & $\begin{array}{c}\text { D2- } \\
\text { D3 }\end{array}$ & $\begin{array}{c}\text { D2- } \\
\text { D4 }\end{array}$ & $\begin{array}{c}\text { D3- } \\
\text { D4 }\end{array}$ & Mean \\
\hline W-CSP & 75.21 & 67.83 & 70.62 & 65.34 & 69.82 & 63.92 & 68.79 \\
\hline $\begin{array}{c}\text { Modified } \\
\text { W-CSP }\end{array}$ & $\mathbf{7 6 . 5 8}$ & $\mathbf{7 2 . 6 3}$ & $\mathbf{7 1 . 5 3}$ & 65.43 & $\mathbf{7 2 . 6 2}$ & $\mathbf{6 8 . 7 0}$ & $\mathbf{7 1 . 2 5}$ \\
\hline
\end{tabular}

TABLE II. PERFORMANCE FOR VARIOUS SUBJECTS IN TERMS OF MEAN CLASSIFICATION ACCURACY (IN PERCENTAGE)

\begin{tabular}{|c|l|c|c|c|c|c|c|}
\hline $\begin{array}{c}\text { Class } \\
\text { versus } \\
\text { Subject }\end{array}$ & $\begin{array}{c}\text { D1- } \\
\text { D2 }\end{array}$ & $\begin{array}{c}\text { D1- } \\
\text { D3 }\end{array}$ & $\begin{array}{c}\text { D1- } \\
\text { D4 }\end{array}$ & $\begin{array}{c}\text { D2- } \\
\text { D3 }\end{array}$ & $\begin{array}{c}\text { D2- } \\
\text { D4 }\end{array}$ & $\begin{array}{c}\text { D3- } \\
\text { D4 }\end{array}$ & Mean \\
\hline S1 & $\mathbf{9 2 . 5}$ & 82.5 & 85 & 77.5 & 81.25 & 85 & 83.96 \\
\hline S2 & 62.5 & 52.5 & 56.25 & 56.25 & $\mathbf{7 1 . 2 5}$ & 57.5 & 59.38 \\
\hline S3 & 81.25 & 76.25 & 72.5 & $\mathbf{8 1 . 2 5}$ & 73.75 & 67.5 & 75.42 \\
\hline S4 & 63.75 & 67.5 & 61.25 & 67.5 & $\mathbf{7 2 . 5}$ & 66.25 & 66.46 \\
\hline S5 & 55 & 53.75 & 55 & 56.25 & $\mathbf{6 0}$ & 58.75 & 56.46 \\
\hline S6 & $\mathbf{8 2 . 5}$ & 80 & 78.75 & 58.75 & 68.75 & 71.25 & 73.33 \\
\hline S7 & $\mathbf{9 8 . 5 7}$ & 95.89 & 91.96 & 60.48 & 80.83 & 74.64 & 83.73 \\
\hline Mean & 76.58 & 72.63 & 71.53 & 65.43 & 72.62 & 68.60 & 71.25 \\
\hline
\end{tabular}

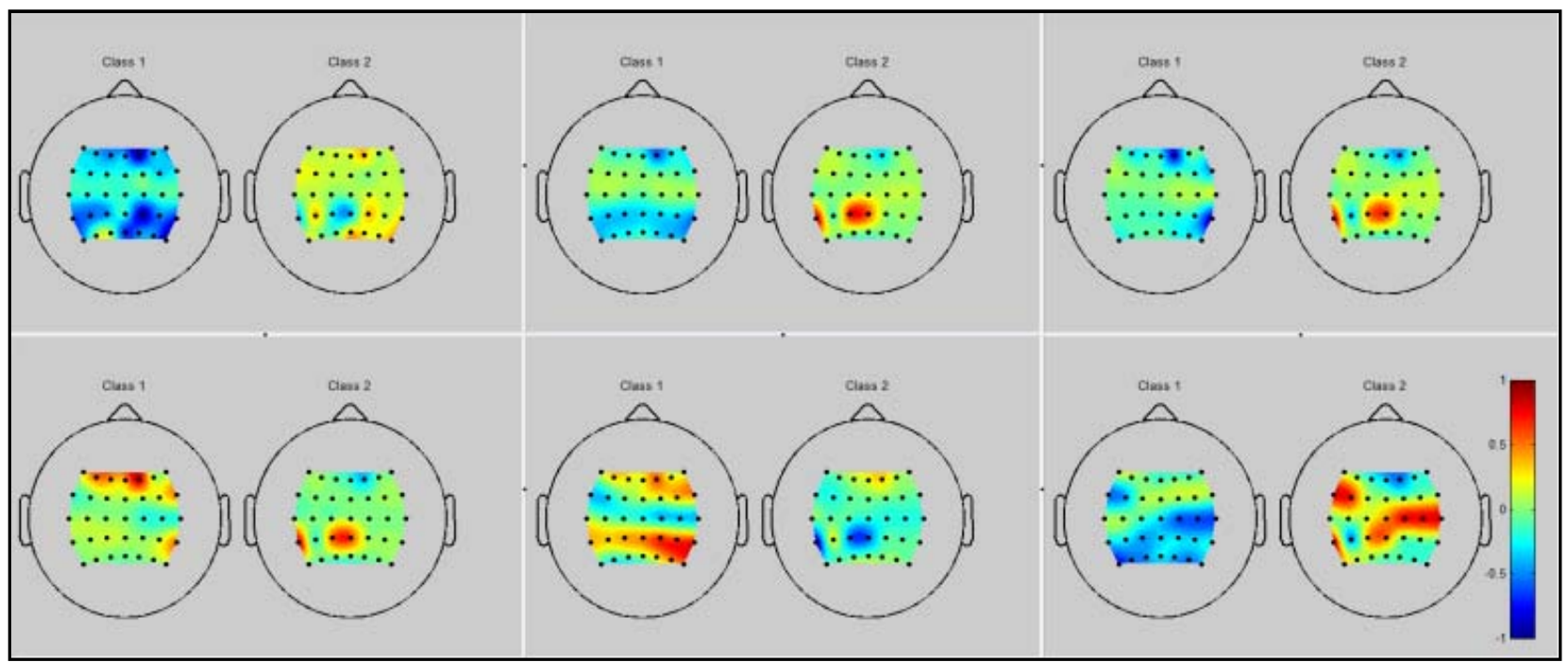

Figure 3. Spatial maps for different subbands obtained from CSP analysis. 